\title{
The role of computer aided design in fashion design teaching exploration
}

\author{
Fengqin Chen ${ }^{1, a}$ \\ ${ }^{1}$ Jiangxi Institute of Fashion Technology, Jiangxi, Nanchang, 330201 \\ a18235291@qq.com
}

Keywords: The computer; Aided design; Clothing design; Colleges and universities

\begin{abstract}
At present, the application of computer technology in teaching has very wide, use of computer technology and multimedia technology assisted teaching has become a clothing teachers in colleges is an important method of teaching. This article first summarizes the merits of the computer aided design, based on the CAD, CORELDRAW, PHOTOSHOP in the clothing design teaching in the important role of the, to the future effective application in the teaching of computer aided design in the clothing design to provide certain reference basis.
\end{abstract}

\section{The introduction}

The join of computer technology and multimedia technology make the costume design teaching from the original traditional teaching gradually to develop in the direction of digitization and automation, CAD and PHOTOSHOP, in the costume design teaching has played the role of nots allow to ignore, not only can better enrich the design technique of expression, but also can make a design more thoroughly the interpretation of the teaching. In order to further promote the effective application of computer aided design in the clothing design teaching, this article mainly several computer aided design software in the role of the costume design teaching in colleges and universities to explore, in order to more effectively improve the quality of teaching.

\section{The advantages of computer aided design}

At present in the teaching of computer aided design in the clothing design reflects the advantages of view, mainly includes two aspects. First of all, using the computer aided design, can greatly improve the working speed and efficiency, but also can effectively avoid the effects of due to human error. In the clothing design process, if use manual operation to solve certain problems, will waste a lot of time. And the high speed processing function of computer have, but can greatly shorten the time. Using computer calculation, the result of the designer to design the depth and breadth of better grasp. For example, in the past time, if students want to draw a piece of clothing renderings, handling of the colors and background can only adopt the way of drawing and print to complete, the overall effect it's very hard to grasp. And since the computer aided design is widely used, students can use PHOTOSHOP software to complete the tonal and background processing, all colors and background simulation can use software to complete, not only can carry on the effective control to the overall effect, and is more convenient to modify and brought a lot of convenience to the designers. At the same time, through the use of all kinds of computer aided design software, designers can put your own design ideas and works more intuitive display through the screen, thus more clearly understand and grasp the content of the design.

Second, can realize the digital design, computer aided design in the past time, almost all of the design is drawn on the paper, the design method is not only difficult to save, and change is difficult. And since the use of computer-aided design, to complete all the drawings are available design software, not only save the storage space, convenient query, but also can carry out remote transmission, realize resources sharing.

\section{Use of garment CAD efficiency}

So-called CAD, mainly refers to the use of computer technology and graphics equipment to help the designers to carry on the design work of the software, at present, has wide application in many 
fields, costume design is one of the most representative. Current clothing design of the entire process, involving many links, such as clothing plate making, model scale, layout, and the fitting and design. In each link is to make full use of CAD software, can be closely integrated with each link of the operation, achieve the goal of automatic production, so as to improve the working efficiency and quality of design. Because of this, CAD software in the current fashion design have got the very extensive application. Colleges and universities as a direct transmission of important place of the senior technology talents to the society, in order to further realize the high technology talented person's raise, must give attaches great importance to the use of garment CAD.

In the clothing design process, the main advantage of the CAD software is able to improve the efficiency of the work of the design, reduce the workload designers, shorten the product production of software in the clothing design advantages of each link, mainly reflected in the following several aspects: (1) application in clothing plate-making process, in the past time, almost all rely on manual to complete, the link and use the CAD, greatly improve the working efficiency, especially in the area of fold change more women's clothing plate making, its efficiency is more than the manual operation. (2) in the clothing retouching link the application of the clothing CAD on has pushed code version as long as the basic code, the other version is automatically changes to the shape, the efficiency is much higher than that of the traditional manual operation. (3) in application of discharge link, can reduce the difficulty of the discharge, let the students can be taught by teachers according to the experience of self discharge, can not only cultivate the students' basic skills, but also can greatly save material and of choose and employ persons.

\section{CORELDRAW in the important role of the costume design teaching in colleges and universities}

CORELDRAW software is commonly used in current fashion design teaching, a computer aided design software. Has the advantage that the software can provide the designer with an extremely detailed drawing and drawing tools, and use method is simple, easy to be accepted by the designers. In recent years, along with Windows in the rising status of software operating platform, CORELDRAW software application in the graphic design is becoming more and more widely.

Auxiliary clothing design using CORELDRAW software, specific to use is very convenient, we take the suit in the design of clothing style mapping as an example, this style of clothing design, the rendering process mainly includes four areas: first, to draw a piece, after and on the basis of the use of graphics capabilities to join rectangles, into curve, then use the plastic tool, on the basis of the specific design requirements for the shape of the piece after adjustment, the link is important to note that in the process of drawing, will come out after fully expressed. Mirror copy after another piece, then copy a as fundamental form of front, welding, after two reuse modelling tools to hem and the radian of neck line for further adjustment of. The above link is completed, the next step is to color fill, under normal circumstances, the color of the filling can be reasonable operation according to the designer's design intention, if the final color effect can't adequately express the intention of the designers, so the software may be used to make changes to its, until satisfied. The last part is the appearance of fabric, in this part, the designer is important to note that the upper and the lower is to belong to two different filling layer, under normal circumstances, the upper is monochromatic filling layer, and part ii is a gradient fill layer, fill of monochromatic moves to the gradient layers, click on the above monochromatic filling layer, and then click the transparent tool, select a bitmap pattern is transparent, the layer properties as normal, choose different transparent tool attribute can produce different fabric texture.

Through the above several parts design, it can be smoothly to suit the design of graph, compared with the traditional manual operation, not only greatly save design time, and modify it more easily. At the same time, also can be the designer's design intent intuitive, comprehensive, for the ascension of the university teaching quality of clothing design is of great significance.

\section{Use PHOTOSHOP to make clothing renderings}

At present, there are two main types of the type of computer drawing graphics, is a kind of vector 
diagram, the other is a lattice figure. Above mentioned all belong to vector graphics, CAD, CORELDRAW software is using geometry formula of performance; And between lattice plan depend on and arrangement to complete, the most representative is the PHOTOSHOP software. In the current fashion design teaching, using PHOTOSHOP software is also very extensive.

In general, use PHOTOSHOP to make clothing renderings, first from the beginning of the line art, drawing for line art designers can by hand, using the software directly, scanning and two ways to complete, PHOTOSHOP to make the application of the clothing renderings, or its processing power of color. In the process of practical application, should be drawn to good sketch lines on the bottom, and based on the layer and the layer using a pencil, brush, paint bucket and the gradient tool fill color according to the requirement, whether flat face, gradients, or color, above all available tools to complete. At the same time, using PHOTOSHOP, designers can also complete the required in the process of drawing a straight line, curve and fuzzy, even can also be defined according to the design intent, it can better rich rendering effect.

PHOTOSHOP software in the teaching of clothing design another advantage is that can use layer styles and filter making unexpected special effects, enhance the visibility of the rendering. In addition, the use of PHOTOSHOP, design also can in the shortest possible time to adjust local color, adjust, designers only need will need to adjust the site selected, fill in color or using the adjustment command and tool change color, is very simple. In a word, use PHOTOSHOP to make clothing renderings, than the traditional manual drawing expression way is simple, specific, effect is good.

\section{Conclusion}

To sum up, computer technology and multimedia technology in the teaching of garment design in the current college occupies the important position, to make full use of the design software, can not only greatly improve the working speed and efficiency, better to grasp the overall design, but also can save the storage space, from the truly realize resources sharing. In the future, therefore, costume design teacher must be combined with the teaching goal, will be reasonably applied to the teaching process of all kinds of design software, and give full play to the role, in order to improve the overall quality of teaching.

\section{Reference}

[1] Hu Yu. The application of computer aided design in art design teaching stage [J]. 2012, 7.

[2] Xiaoyan Zhang. The application of computer aided design in architectural design teaching [J] China forestry education. 2009, 7.

[3] Zhou Jia. The role of computer aided design in art and design curriculum system [J] mass artistic analysis and teaching thinking. 2009, 7. 